\title{
EDITORIAL
}

\section{Bacteriophages, revitalized after 100 years in the shadow of antibiotics}

\author{
Hongping Wei ${ }^{\otimes}$ \\ Wuhan Institute of Virology, Chinese Academy of Sciences, Wuhan 430071, China
}

The year 2015 marks 100 years since Dr. Frederick Twort discovered the "filterable lytic factor", which was later independently discovered and named "bacteriophage" by Dr. Felix d'Herelle. On this memorable centennial, it is exciting to see a special issue published by Virologica Sinica on Phages and Therapy. In this issue, readers will not only find that bacteriophage research is a booming field but also learn about the diverse applications currently being explored for bacteriophages. The biggest driving force behind these applications is the serious threat of bacterial antibiotic resistance that is emerging in the current era (Figure 1).

In 2014, we witnessed US president Barack Obama announced a comprehensive set of new federal initiatives to combat the rise of antibiotic-resistant bacteria and protect public health. As stated in the first global report on antibiotic resistance, "Antimicrobial Resistance Global Report 2014" released by the World Health Organization (WHO), it is clear that "A post-antibiotic era - in which common infections and minor injuries can kill - far from being an apocalyptic fantasy, is instead a very real possibility for the twenty-first century" (Keiji Fukuda, WHO Assistant Director-general for Health Security, in a foreword to the report).

Why would a post-antibiotic era be so horrible for humans? Antimicrobial drugs not only have revolutionized the way we treat patients with bacterial infections but are also one of the pillars of modern medicine. Without effective antibiotics, many medical procedures, such as surgical operations and transplantations, will be fatal due to the high risk of infections. At such a critical time, it is urgent that all stakeholders take drastic actions to stop antimicrobial resistance.

As natural biocides of bacteria, there is no doubt that bacteriophages are returning to the spotlight after 100 years in the shadows. Because bacteriophages evolve faster than the bacteria they infect, they have a distinct advantage over antibiotics in overcoming bacterial resistance. New bacteriophages that are effective against resistant bacteria can be isolated in days or weeks at a very modest cost. In contrast, the development of a new antibiotic takes years and costs hundreds of millions of dollars. Bacteriophages are also safe as underscored by their extensive clinical use in Eastern Europe and the former Soviet Union as well as the commercial sale of bacteriophages during the $1940 \mathrm{~s}$ in the United States.

This special issue includes studies on several newly discovered phages. The novel lytic cold-active bacteriophageVMY22 of Bacillus cereus (Ji et al., 2015) and the novel transposable Mu-like prophage BalMu-1 of Bacillus alcalophilus (Yang et al., 2015), which reveal the broad diver- sity of bacteriophages and demonstrate that new biological functions can be discovered by further studies of these phages. The isolation and characterization of bacteriophages against extended spectrum $\beta$-lactamase (ESBL)-producing Escherichia coli (Abdulkarim et al., 2015), Klebsiella pneumonia (Lu et al., 2015), and mycobacteria (Teng et al., 2015) add new members to the phage family, which could be important for formulating phage cocktails to control those bacteria species that are known for antimicrobial resistance. However, since not every isolated bacteriophage is suitable for phage therapy, the study by Victor Krylov (Krylov et al., 2015) offers some important considerations for the

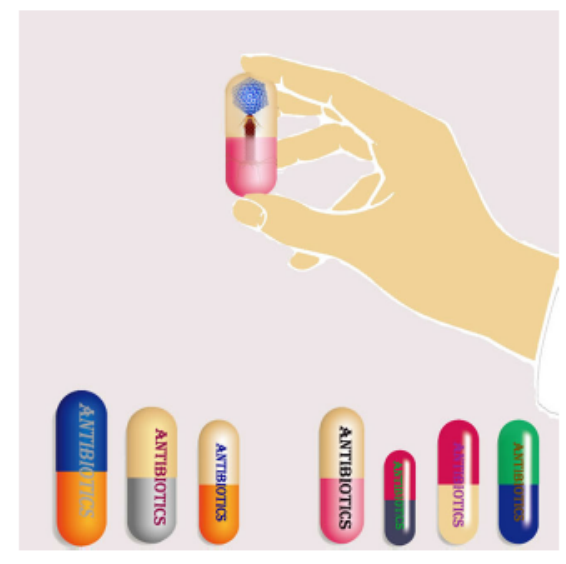

Figure 1. As natural biocides of bacteria, bacteriophages are returning to the spotlight after 100 years discovery. (1915-2015) 
selection of phages and the criteria required for safe phage therapy.

The interactions between bacteriophages and their hosts are complicated but important for both phage biology and phage applications. The review by Dr. Stephen Abedon (Abedon, 2015) describes different types of bacteriophage secondary infections and the importance of clarification when using the term. Another review by Fan and Kan (Fan and Kan, 2015) summarizes the survival and proliferation of the important lysogenic bacteriophage СТХФ in Vibrio cholerae. Because phage resistance and infectivity are important factors to consider during phage therapy, the study on the variation of the resistance and infectivity between Pseudomonas fluorescens SBW25 and bacteriophage $\Phi 2$ (Chen and Chen, 2015) demonstrates how phage resistance and infectivity might be affected during its interaction with the host.

In addition to these studies on bacteriophages, in recent years, the lytic proteins expressed by bacteriophages are also being actively explored for their bactericidal abilities. Generally, recombinant proteins are much simpler than bacteriophages; therefore, recombinant lytic proteins may be easier to control and might be more suitable for use as therapeutic drugs. The recombinant lyase gp17 (Wang et al., 2015) was shown to inhibit the growth of E. coli 8401 . Molecular dissection of the wide antimicrobial spectrum lysin PlySs2 revealed some unique properties of its cell wall binding and catalytic domains against Streptococci and
Staphylococci (Yang et al., 2015). The review by David Trudil (Trudil, 2015) provides an excellent summary of the early history of bacteriophage lysins and their applications in various aspects.

Last but not least, the insight article by Mzia Kutateladze (Kutateladze, 2015) reports the successful bacteriophage therapy experience of the world-famous Eliava Institute, which definitely reinforces our confidence in treating clinical bacterial infections using bacteriophages. The review by $\mathrm{Xu}$ et al. (2015) summarizes recent progress in bacteriophage therapy against Enterobacteriaceae. Carbapenemresistant Enterobacteriaceae have attracted much attention in recent years because they are difficult to treat due to their high levels of resistance to antibiotics.

One hundred years have passed since the discovery of bacteriophages. It seems ironic that antibiotic resistance is pushing us back into the pre-antibiotic era when bacteriophages were first discovered. Fortunately, with all the experience and knowledge we have obtained, there is great optimism that the reintroduction of bacteriophage therapy will help to save the human race from returning to the pre-antibiotic era. It is hoped that further studies on bacteriophages and their successful applications emerge in the future.

\section{FOOTNOTES}

I would like to thank all authors for their excellent contributions to this special issue, and the editorial office of Vrologica Sinica for their great support. Many other people have helped to make this special issue a reality: Prof. Ran Wang, Prof. Yigang Tong, Prof. Fuquan $\mathrm{Hu}$, and Dr. Sixiang Zhou participated in the article invitation for this special issue; Dr. Junping Yu and Dr. Hang Yang helped with the issue coordination. I want to thank all of them for their great contribution.

This work was supported by the Basic Research Program of the Ministry of Science and Technology of China (2012CB721102), the Chinese Academy of Sciences (grant No: KJZD-EW-L02), and the Key Laboratory on Emerging Infectious Diseases and Biosafety in Wuhan.

\author{
$\triangle$ Correspondence: \\ Phone: +86-27-51861076, \\ Fax: +86-27-87199492, \\ Email: hpwei@wh.iov.cn \\ ORCID:0000-0002-9948-8880
}

Published online: 5 February 2015

\section{REFERENCES}

Abedon ST. 2015. Virol Sin, 30:3-10.

Ahmad KA, Mohanmmed AS, Abas F, et al. 2015. Virol Sin, 30:73-75.

Chen HC, Chen GH. 2015. Virol Sin, 30:59-62.

Fan FX, Kan B. 2015. Virol Sin, 30:19-25.

Ji XI, Zhang CJ, Fang Y, et al. 2015. Virol Sin, 30:52-58.

Krylov V, Shaburova O, Pleteneva E, et al. 2015. Virol Sin, 30:33-44.

Kutateladze M. 2015. Virol Sin, 30:80-81.

Lu YY, Shi HY, Zhang Z, et al. 2015. Virol Sin, 30:66-68.

Teng TS, Yu JP, Yang H, et al. Virol Sin, 30:76-79.

Trudil D. 2015. Virol Sin, 30:26-32.

Wang TW, Lin H, Zhang L, et al. 2015. Virol Sin, 30:69-72.

Xu YQ, Liu Y, Liu Y, et al. 2015. Virol Sin, 30:11-18.

Yang HL, Yang H, Yu JP, et al. 2015. Virol Sin, 30:45-46.

Yang JJ, Kong YM, Li X, et al. 2015. Virol Sin, 30:63-65. 